\title{
MINDFULNESS PARA LA REDUCCIÓN DEL MALESTAR EMOCIONAL EN PACIENTES ONCOLÓGICOS. ESTUDIO COMPARATIVO CON UNA INTERVENCIÓN PSICOEDUCATIVA ESTÁNDAR
}

\author{
EFFECTIVENESS OF A MINDFULNESS-BASED STRESS REDUCTION (MBSR) \\ INTERVENTION VERSUS A PSYCHOEDUCATIONAL STANDARD INTERVENTION IN \\ PATIENTS WITH CANCER
}

\author{
Concha León', Rosanna Mirapeix', Tomás Blasco², Esther Jovell³, Àngels Arcusa ${ }^{4}$, Andrés \\ Martín ${ }^{5}$ y Miquel Àngel Seguí \\ I Institut Oncològic del Vallès (Consorci Sanitari de Terrasa-Corporació Sanitària Parc Tauli) \\ 2 Departament de Psicologia Bàsica. Universitat Autònoma de Barcelona \\ 3 Unidad de Epidemiología y Evaluación Asistencial. Consorci Sanitari de Terrassa \\ ${ }^{4}$ Consorci Sanitari de Terrassa. Institut Oncológic del Vallès \\ 5 INSPIRA Desarrollo Personal y Aprendizgje Organizativo \\ 6 Hospital Parc tauli de Sabadell. Institut Oncológic \\ del Vallès
}

Resumen

Objetivo: Comparar la efectividad de una intervención de Mindfulness (MBSR) versus una intervención psicoeducativa estándar en pacientes oncológicos.

Método: La intervención MBSR (grupo experimental) consistió en 10 sesiones semanales de 2,5 horas cada una de ellas y el paciente debía practicar Mindfulness diariamente y durante 45 minutos en su casa y la intervención psicoeducativa (grupo control) consistió en 8 sesiones semanales de 2,5 horas. Participaron 20 y 17 personas, respectivamente. Se recogieron datos basales y se administraron (pretestpostest) los siguientes cuestionarios a los participantes de ambos grupos: STAI (ansiedadestado y ansiedad-rasgo), BDI (depresión) y EORT-QLQ C-30 (calidad de vida).

Resultados: En el grupo experimental resultaron diferencias estadísticamente significativas indicativas de mejoría en todas las medidas (depresión, ansiedad-estado, ansiedad-rasgo y calidad de vida) mientras que en el grupo de control sólo aparecieron en la variable ansiedad-estado.

Conclusiones: Los datos de esta investigación indican que el MBSR produce una me-
Abstract

Purpose: To compare the effectiveness of a Mindfulness-Based Stress Reduction (MBSR) intervention versus a psychoeducational standard intervention in oncological patients.

Method: MBSR intervention was applied following a 2'5 h-session schedule, across 10 weekly sessions. Patients $(n=20)$ had to practice mindfulness daily at home during $45 \mathrm{mi}-$ nutes. In the psychoeducational group $(n=17)$ a schedule of 8 weeekly sessions of $2^{\prime} 5$ h each was applied. Following a pre-post design, the following questionnaires were applied to both groups of patients: STAI (to assess both state and trait anxiety), BDI (Depression) and EORTC-QLQ-C-30 (quality of life).

Results: In the MBSR group, scores in anxiety and depression decreased at post-treatment assessment whereas quality of life scores increased. These differences reached statistical significance. In the psychoeducational group only state anxiety scores decreased at posttreatment, with the difference reaching statistical significance.

Conclusions: MBSR produces higher improvements in emotional states and quality of

Correspondencia:

Tomás Blasco Blasco

Departament de Psicologia Bàsica, Evolutiva i de l'Educació. Universitat Autònoma de Barcelona. 08193 Bellaterra (Barcelona) E-mail: tomas.blasco@uab.es 
joría importante en el malestar emocional y en la calidad de vida, superior a la que puede lograrse con una intervención psicoeducativa. No obstante, no puede afirmarse que el MBSR deba ser la técnica de elección para todos los casos, pues no está claro que pueda ser seguida por cualquier tipo de paciente, al requerir una alta implicación por parte del mismo.

Palabras Clave: Mindfulness, MBSR, calidad de vida, ansiedad, depresión, cáncer. life than those obtained following a psychoeducational standard procedure. However, it cannot be stated that MBSR must be always the intervention to be applied in all cases, since it is not clear that all patients could follow the guidelines, tasks and exercices required by this technique.

Key words: Mindfulness, MBSR, quality of life, anxiety, depression, cancer.

\section{INTRODUCCION}

El cáncer es una enfermedad frecuente y compleja que compromete la supervivencia de la persona que la padece $y$, por extensión, genera un estrés socioemocional $^{(1,2)}$ que condiciona su calidad de vida y justifica la demanda de atención psicoterapéutica y psicoeducativa como complemento al tratamiento médico que se recibe. Bajo esta perspectiva, se han desarrollado diferentes intervenciones psicosociales que han demostrado ampliamente su eficacia en la mejora de las habilidades de afrontamiento, la calidad de vida, la reducción del malestar emocional y la sensación de aislamiento en pacientes oncológicos $^{(3-5)}$.

Dentro de estas estrategias, el Mindfulness-Based Stress Reduction (MBSR) es un tipo de intervención terapéutica desarroIlada por Jon Kabat-Zinn ${ }^{(6)}$ que consiste, tal como describen Martín-Asuero y GarcíaBanda (7), en "la intención sostenida de centrar la atención en la realidad del momento presente, aceptándola sin juicios y sin dejarse llevar por los pensamientos ni por las reacciones emotivas del momento" y, por tanto, "requiere mantener la atención en lo que ocurre aquí y ahora". Los programas de MBSR contemplan, habitualmente, entre 7 y 10 sesiones grupales de 1,5 o 2,5 horas de duración y tienen un claro enfoque hacía la interacción cuerpo-mente, alternando la práctica de ejercicios corporales y estiramientos tipo yoga con la meditación y potenciando la atención plena durante la realización de las actividades cotidianas. Numerosos estudios evidencian la eficacia de este tipo de intervenciones en el ámbito de la salud. De hecho antes de la década de los 90 ya existía literatura que demostraba, no sin señalar ciertas limitaciones metodológicas, su utilidad en pacientes con dolor crónico ${ }^{(8)}$, y más adelante se encuentran evidencias en pacientes con trastornos de ansiedad ${ }^{(9,10)}$, fibromialgia ${ }^{(11)}$, epilepsia $^{(12)}$, hipertensión ${ }^{(13)}$ y psoriasis ${ }^{(14)}$. Un trabajo de meta-análisis ${ }^{(15)}$ señala un efecto claro, aunque moderado, del MBSR en pacientes con diferentes problemas de salud.

En cuanto a la aplicabilidad clínica del MBSR en pacientes oncológicos puede considerarse como pionero el estudio realizado por Carlson y Speca en el año $2000^{(16)}$. Desde entonces, han sido numerosos los trabajos que han evaluado la eficacia del MBSR en población oncológica, quedando ésta avalada tanto a través de meta-análisis ${ }^{(17)}$ como mediante una revisión crítica ${ }^{(18,19)}$.

Los efectos beneficiosos se han constatado en pacientes que atravesaban la fase de tratamiento y en diferentes aspectos psicológicos como el estrés y la rumiación ${ }^{(20,21)}$. Respecto a los aspectos físicos, se ha observado mejoría en la fatiga ${ }^{(22)}$, pero no en la presión sanguínea ${ }^{(21)}$. La reducción del estrés también se ha observado en las parejas de los pacientes que participaron en el programa ${ }^{(20)}$. El MBSR ha 
mostrado también su eficacia para reducir el malestar emocional en supervivientes de cáncer ${ }^{(23,24,33)}$ y se ha sugerido que esta mejoría podría deberse a que el MBSR facilitaría el crecimiento personal ${ }^{(25)}$.

A pesar de estas evidencias, quedan cuestiones por resolver respecto a cuál es el grado de eficacia concreto que puede obtenerse aplicando el MBSR a pacientes oncológicos, puesto que los trabajos de revisión coinciden en señalar que hay bastantes limitaciones metodológicas en las investigaciones publicadas y que si el meta-análisis incluye únicamente los artículos que utilizan diseño aleatorizado, el tamaño del efecto se reduce sensiblemente $^{(26)}$. Así, algunas investigaciones no utilizan la aleatorización para construir el grupo control ${ }^{(27)}$ o aplican únicamente un diseño pre-post ${ }^{(20,28)}$, mientras que otras, si bien utilizan un grupo control aleatorizado, éste no recibe tratamiento, manteniéndose en lista de espera o sin recibir tratamiento específico ${ }^{(21,23,29-31)}$ por lo que es difícil concluir si el MBSR, aun siendo aparentemente eficaz, ofrece mejores resultados que otros tipos de intervención.

No obstante, han empezado a aparecer trabajos que sí han utilizado la metodología de grupo control aleatorizado en el que éste recibe tratamiento activo. Concretamente, Garland et al. ${ }^{(32)}$, lo han aplicado para estudiar la eficacia del MBSR sobre el insomnio en pacientes supervivientes, comparándola con la terapia cognitivoconductual. Carlson et al. 2013(33), también en pacientes supervivientes, han observado una mayor eficacia del MBSR mediante un diseño aleatorizado en que se comparaba a los pacientes que recibían este tratamiento con otros dos grupos (uno que recibió terapia grupal de apoyo emocional, y otro que recibió una condición control basada en un asesoramiento sobre manejo del estrés). Por su parte, Henderson et al. ${ }^{(34)}$ han evaluado el efecto del MBSR sobre la calidad de vida en pacientes con cáncer de mama, pero el grupo de comparación no ha recibido un tratamiento dirigido a los aspectos psicológicos, sino que ha sido remitido a un programa de educación nutricional.

Está claro, pues, que para conocer mejor cuál es el grado de eficacia del MBSR sobre diferentes indicadores del bienestar y la calidad de vida de pacientes de cáncer (durante las diferentes fases de la enfermedad o en la supervivencia) es necesario desarrollar estudios que utilicen diseños aleatorizados en los que el grupo control reciba una intervención en la que se aplique alguna técnica cuya eficacia haya sido ya previamente demostrada. El presente trabajo sigue esta metodología y pretende comparar la efectividad de dos intervenciones grupales, una basada en el MBSR y otra psicoeducativa estándar para la reducción del malestar psicológico asociado al estrés en pacientes de cáncer tanto si se encuentran en situación de tratamiento activo como si han finalizado el mismo.

\section{MÉTODO}

\section{Diseño}

Estudio exploratorio cuasiexperimental de comparación de dos grupos equivalentes. El estudio se realizó en el Institut Oncològic del Vallès (IOV), entidad de la provincia de Barcelona, que presta asistencia a los pacientes oncológicos del Hospital Parc Taulí de Sabadell y del Hospital de Terrassa que cuentan con una población de referencia de 600.000 personas. Los pacientes de uno de los hospitales recibieron el tratamiento basado en el programa MBSR, mientras que los del otro hospital recibieron el programa psicoeducativo.

\section{Participantes}

El estudio incluyó dos grupos de pacientes diagnosticados de cáncer, mayo- 
res de 18 años y previa firma del consentimiento informado. Se incluyó tanto a pacientes que estaban en tratamiento activo como a pacientes que ya lo habían finalizado. Los criterios de exclusión considerados fueron: analfabetismo, trastorno psíquico que impidiese la comprensión de las instrucciones e incapacidad física.

\section{Materiales y Procedimiento}

La captación de los sujetos se realizó mediante carteles divulgativos colocados en las consultas de Oncología y la derivación por parte de las psicooncólogas y oncólogos de ambos hospitales, después de un primer contacto con cada paciente donde se le informaba de las características del estudio y se les invitaba a participar.

La intervención en el grupo experimental siguió el programa MBSR tal y como lo describen Martin-Asuero y Garcia de la Banda $^{(7)}$ siguiendo el modelo de Kabat$Z_{\text {Zinn }}{ }^{(6)}$. Fue conducida por un instructor formado en el Centro Médico de Reducción del Estrés de la Universidad de Massachusetts (EEUU) con la colaboración de una psicooncóloga. El formato de la intervención consistió en 10 sesiones semanales de 2,5 horas cada una. Cada sesión incluía la presentación de un tema, ejercicios prácticos y un debate en grupo. El entrenamiento, a través de la práctica de ejercicios de focalización de la atención, meditación y estiramientos tipo yoga, pretendía el desarrollo de la Conciencia Plena en las actividades cotidianas. Durante el programa se explicó cómo afrontar las emociones negativas, el estrés y el dolor asociados a la enfermedad. Asimismo, las sesiones contemplaron un tiempo para el debate en grupo sobre los aciertos y las dificultades que surgían al aplicar la técnica en la vida diaria dado que tenían que practicarla durante aproximadamente 45 minutos al día, para lo que se les facilitó un juego de 3 CDs de audio y documentación con lecturas y ejercicios.

La intervención en el grupo control fue conducida por una psicooncóloga y siguió un programa psicoeducativo, de manera que, en cada sesión, la terapeuta aportaba información psicoeducativa sobre un tema en concreto (autoestima, ansiedad, sexualidad, comunicación, estrategias de afrontamiento, etc.), y seguidamente éste se trabajaba en base a diferentes técnicas cognitivo-conductuales promoviendo la participación activa de todos los participantes y el debate grupal. El formato de la intervención consistió en 8 sesiones semanales de 2,5 horas cada una.

\section{Mediciones}

Se recogieron datos basales (datos sociodemográficos, datos clínicos de la enfermedad, tipo de cáncer, fecha del diagnóstico, estadio y extensión de la enfermedad, tratamientos farmacológicos y terapias complementarias y/o alternativas), y se administraron, antes de que empezase la intervención y el día en que ésta finalizó, los siguientes cuestionarios a los sujetos de ambos grupos:

- Cuestionario de ansiedad (STAI StateTrait Anxiety Inventory) ${ }^{(35)}$. Consta de 2 escalas, de 20 ítems cada una, que miden facetas de la ansiedad diferentes pero relacionadas entre ellas: estado (STAI-E) y rasgo (STAI-R).

- Cuestionario de depresión (BDI IIBeck Depression Inventory) $)^{(36)}$. Es un cuestionario autoaplicado de 21 ítems que evalúa un amplio espectro de síntomas depresivos y ha sido validado para la población española ${ }^{(37)}$. Los baremos establecen que una puntuación entre 0 y 9 indica ausencia de depresión, una puntuación entre 10 y 18 , una depresión leve, una puntuación entre 19 y 29, una depresión mode- 
rada, y una puntuación entre 30 y 63, una depresión grave.

- Cuestionario de calidad de vida (EORTC-QLQ C-30-European Organization for Research and Treatment of Cancer) ${ }^{(38)}$. Consta de 30 ítems que incluyen 5 áreas funcionales (física, emocional, cognitiva, social y funcional), 3 escalas de síntomas (fatiga, dolor, náuseas y vómitos), una escala global de calidad de vida y 6 ítems sobre síntomas comunes (disnea, alteraciones del sueño, apetito, estreñimiento, diarrea y problemas económicos).El cuestionario ha sido validado para la población española(39) y en esta investigación se utilizó únicamente la escala global de calidad de vida en la que una mayor puntuación indica un mayor nivel de calidad de vida.

\section{ANÁLISIS ESTADÍSTICO}

Para todas las variables recogidas se realizaron los estadísticos descriptivos más comunes. En el caso de las variables nominales se presentaron los porcentajes (\%) de cada una de las categorías previamente definidas. Para las variables discretas se determinaron la media y la desviación estándar.

Se valoró la equivalencia de los grupos respecto a las características basales (edad, sexo, situación laboral, convivencia, nivel de estudios, situación clínica) y a las puntuaciones en los cuestionarios de calidad de vida, STAI-E, STAI-R, y BDI-II mediante pruebas de ji cuadrado o de comparación de medias.

Para la comparación de las determinaciones pre y post de los cuestionarios se utilizó la prueba estadística t de Student para datos apareados. El nivel de significación estadístico considerado fue del 5\% y el paquete estadístico utilizado para el análisis fue el Statistical Package for the Social Sciences (SPSS) versión 20.0.

\section{RESULTADOS}

De los 37 participantes (20 en el grupo experimental y 17 en el grupo control) que iniciaron el estudio, completaron la intervención 27, 16 del grupo experimental y 11 del grupo control. De los que aceptaron participar en el estudio, un 83,8\% (31) eran mujeres, la media de edad fue de 52 años (DT:11) (rango: 27-77), la neoplasia diagnosticada con más frecuencia en 17(46\%), fue el cáncer de mama. En nuestra muestra, 21(57\%) no trabajaban, $21(57 \%)$ habían cursado estudios medios y $33(83 \%)$ vivían solos.

En la tabla 1 se muestran las características basales de los participantes que completaron la intervención. No se encontraron diferencias estadísticamente significativas entre grupos en cuanto a edad, sexo, vivir solo, situación laboral actual, recibir tratamiento activo, BDI-II basal, STAI-E y STAI-R basales y la puntuación global basal de calidad de vida.

En la tabla 2 se indican los valores obtenidos antes y después de la intervención para cada una de las variables en cada uno de los grupos del estudio. Hay diferencias estadísticamente significativas en todas ellas en el grupo experimental, mientras que en el grupo control sólo las hay para la dimensión STAI-E ( $p=0,027)$.

\section{DISCUSIÓN}

Los resultados indican que los pacientes que siguieron la intervención basada en Mindfulness, redujeron sus puntuaciones en los cuestionarios que evaluaban aspectos emocionales, y aumentaron la de la calidad de vida, al igual que en otras investigaciones realizadas tanto en supervivientes como en pacientes en situación de tratamiento ${ }^{(20,23,33)}$. Por lo tanto, lo observado en la presente investigación está de acuerdo con lo que cabía esperar habida cuenta del conocimiento disponible 
Tabla 1. Características basales de los participantes en ambos grupos

\begin{tabular}{|c|c|c|c|}
\hline & $\begin{array}{l}\text { Grupo experimental } \\
\qquad \begin{array}{c}\mathrm{N}=20 \\
\mathrm{n}(\%)\end{array}\end{array}$ & $\begin{array}{l}\text { Grupo control } \\
\qquad \begin{array}{c}\mathrm{N}=17 \\
\mathrm{n}(\%)\end{array}\end{array}$ & p-valor \\
\hline Sexo (mujer) & $16(80)$ & $15(88,2)$ & 0,413 \\
\hline $\begin{array}{l}\text { Nivel de educación: } \\
\text { - no estudios, si leer y } \\
\text { escribir } \\
\text { - primarios } \\
\text { - medios } \\
\text { - universitarios }\end{array}$ & $\begin{array}{l}1(5) \\
4(20) \\
11(5) \\
4(20)\end{array}$ & $\begin{array}{c}5(29,4) \\
10(58,8) \\
2(11,8)\end{array}$ & \\
\hline $\begin{array}{l}\text { Situación laboral actual: } \\
\text { - no trabajo } \\
\text { - activo }\end{array}$ & $\begin{array}{c}12(60) \\
8(40)\end{array}$ & $\begin{array}{l}9(52,9) \\
8(47,1)\end{array}$ & 0,460 \\
\hline $\begin{array}{l}\text { Vive sólo: } \\
\text { - no } \\
- \text { si }\end{array}$ & $\begin{array}{c}17(85) \\
3(15)\end{array}$ & $\begin{array}{c}16(94,1) \\
1(5,9)\end{array}$ & 0,367 \\
\hline $\begin{array}{l}\text { Tipo de cáncer: } \\
\text { - glándula salival } \\
\text { - próstata } \\
\text { - colon } \\
\text { - linfático } \\
\text { - mama } \\
\text { - astrocitoma } \\
\text { - cavum } \\
\text { - linfoma inguinal } \\
\text { - pulmón } \\
\text { - ovario } \\
\text { - esófago } \\
\text { - otros }\end{array}$ & $\begin{array}{c}1(5) \\
1(5) \\
7(35) \\
1(5) \\
8(40) \\
1(5) \\
\\
1(5)\end{array}$ & $\begin{array}{l}2(11,8) \\
9(52,8) \\
1(5,9) \\
1(5,9) \\
1(5,9) \\
1(5,9) \\
1(5,9) \\
1(5,9)\end{array}$ & \\
\hline \multirow[t]{2}{*}{$\begin{array}{l}\text { Situación clínica } \\
\text { - En tratamiento activo } \\
\text { - Sin tratamiento activo }\end{array}$} & $\begin{array}{c}8(40) \\
12(60)\end{array}$ & $\begin{array}{c}10(58,8) \\
7(41,2)\end{array}$ & 0,209 \\
\hline & Media(DE) & Media(DE) & p-valor \\
\hline Edad & $52(13)$ & $52(6)$ & 0,969 \\
\hline BDI-II & $24,35(13,8)$ & $19,06(8,77)$ & 0,182 \\
\hline STAI - E & $32,75(14,1)$ & $25,41(11,34)$ & 0,095 \\
\hline STAI - R & $31,05(14,7)$ & $28,18(8,45)$ & 0,483 \\
\hline EORTC-QLQ C-30 Global & $48,33(25,59)$ & $56,86(26,71)$ & 0,329 \\
\hline
\end{tabular}


Tabla 2. Valores de las medidas de depresión, ansiedad y calidad de vida antes y después de la intervención.

\begin{tabular}{|l|c|c|c|}
\hline \multicolumn{1}{|c|}{ GRUPO } & $\begin{array}{c}\text { PRE- INTERVENCIÓN } \\
\text { Media (DE) }\end{array}$ & $\begin{array}{c}\text { POST- INTERVENCIÓN } \\
\text { Media (DE) }\end{array}$ & p-valor \\
\hline EXPERIMENTAL & $24,00(15,03)$ & & \\
\hline BDI & $30,63(14,71)$ & $16,20(9,57)$ & $<0,001$ \\
STAI - E & $28,75(14,53)$ & $19,44(13,24)$ & 0,001 \\
STAI - R & $53,88(21,79)$ & $73,88(18,59)$ & 0,001 \\
EORTC-QLQ C-30 Global & & & 0,011 \\
\hline CONTROL & $18,55(10,18)$ & $16,64(12,22)$ & \\
BDI & $24,27(12,82)$ & $19,45(12,92)$ & 0,312 \\
STAI - E & $27,55(9,59)$ & $28,64(12,15)$ & 0,027 \\
STAI - R & $56,06(26,37)$ & $68,18(25,22)$ & 0,096 \\
EORTC-QLQ C-30 Global & &
\end{tabular}

sobre los beneficios del MBSR que se han descrito en la introducción. La aportación que proporciona el presente estudio es la de mostrar que la intervención basada en el MBSR es más eficaz que la basada en una intervención psicoeducativa y ello independientemente de que los pacientes estuvieran recibiendo tratamiento activo o no, ya que los pacientes del grupo control sólo redujeron sus puntuaciones en una de las escalas de valoración de aspectos emocionales. Ahora bien, estas consideraciones deben ser matizadas.

En primer lugar, no debe olvidarse que el presente estudio tiene una muestra pequeña y heterogénea, y ello reduce tanto a la validez externa, como la potencia estadística. Respecto a la primera limitación, cabe señalar que no es motivo para desestimar los resultados, ya que existen trabajos en los que el tamaño de los grupos también es inferior a 30 participantes ${ }^{(21,40)}$. El que la muestra no sea homogénea respecto al hecho de recibir o no tratamiento activo es un aspecto importante, pero, dado que los grupos han quedado equilibrados respecto a esta variable, creemos que los datos permiten sugerir que este factor no afecta a la eficacia del MBSR.
Respecto a la baja potencia estadística, el que se hayan encontrado diferencias en el grupo experimental en todas las variables indica que no puede descartarse que el MBSR mejore el estado de los pacientes con mayor magnitud que una intervención psicoeducativa.

En segundo lugar, la reducción de puntuaciones en depresión en el grupo experimental es muy espectacular, ya que los pacientes pasan, en promedio, de unas puntuaciones que les sitúan en niveles de depresión moderada a valores que indican prácticamente ausencia de depresión. En cambio, en el grupo control, las puntuaciones se mantienen en niveles de depresión leve. Es difícil cotejar estos resultados pues en ninguna de las investigaciones consultadas se ha utilizado el BDI. Es posible que la reducción sea similar a la que se ha observado en trabajos que han utilizado el POMS ${ }^{(20,31,33)}$. Tampoco puede descartarse que el grupo experimental fuera más receptivo a los efectos del tratamiento por tener, al inicio, unas puntuaciones en depresión superiores a los del otro grupo, aun cuando, tal como hemos indicado en la tabla 1, los grupos fueran estadísticamente equivalentes. En cualquier caso, y 
de acuerdo con los objetivos de nuestro trabajo, lo importante es destacar que esta reducción no se ha observado en los pacientes que recibían intervención psicoeducativa, por lo que debe considerarse que, a falta de trabajos que repliquen nuestra investigación y lo confirmen, el MBSR parece ser una estrategia más eficaz para reducir el estado de ánimo depresivo.

Un tercer aspecto que hay que analizar con más detalle es el cambio en los indicadores de ansiedad. Si bien es esperable una reducción en la ansiedad-estado, y que ésta se consigue con ambos tipos de intervención, la reducción es mayor en el grupo experimental, siguiendo así el mismo perfil que el observado en las puntuaciones de depresión, por lo que podríamos considerar de nuevo la posibilidad de que el hecho de partir con niveles más elevados de alteración emocional puede hacer que los pacientes tengan un margen de mejoría superior al recibir una intervención psicológica. Los cambios experimentados en la ansiedad-rasgo, que sólo se dan en el grupo experimental, entran en contradicción con la supuesta estabilidad de un aspecto de la personalidad como el rasgo. Es posible que se esté produciendo algún tipo de sesgo en la valoración que hace el paciente cuando responde los ítems y que evalúe sólo las últimas semanas al responder a la instrucción "cómo se siente usted, en general", en lugar de hacerlo respecto a un espacio de tiempo más amplio. De ser así, lo que estaría indicando esta medida es que el paciente considera que, globalmente, su estado de ansiedad es más bajo que el que tenía antes de recibir la intervención. En todo caso, queda por explicar por qué ese supuesto sesgo se da sólo en el grupo experimental y no aparece en el grupo control. Una vez más, puede apuntarse a que una posible explicación es que el sesgo es causado por la propia sensación de mejoría que parecen tener los pacientes del grupo experi- mental Finalmente, la mejoría en el grupo experimental en la calidad de vida corrobora la mejora del estado emocional que se observa en los indicadores antes comentados, al igual que se ha observado en supervivientes ${ }^{(23,33)}$. Lo interesante de este dato es que, una vez más, esos cambios en las puntuaciones no se dan en el grupo que recibe la intervención psicoeducativa, lo que reafirma la idea de que, aun con las limitaciones metodológicas de nuestro trabajo, el MBSR parece ser un tipo de intervención más eficaz.

Podemos considerar, pues, que nuestros resultados van en la misma línea que otros trabajos que han mostrado la mayor eficacia del MBSR respecto a la no intervención basada en listas de espera ${ }^{(21)}$ o a los cuidados habituales sin intervención ${ }^{(23)}$, aportando evidencia a favor de que, además, el MBSR es más eficaz que una intervención psicoeducativa e independientemente de que los pacientes se encuentren en situación de tratamiento activo o no. Esto hace que podamos pensar que el MBSR debería ser la técnica de elección. Sin embargo, y desde un punto de vista más aplicado, hay que ser cautelosos, ya que si bien los datos sugieren que los pacientes se benefician de la técnica, no es menos cierto que ésta requiere una alta implicación y dedicación de los enfermos y esto es difícil de conseguir, sobre todo cuando estos se encuentran en la fase de tratamiento, con las limitaciones y problemas que el mismo suele conllevar. Los cuatro abandonos en el grupo de Mindfulness de nuestra investigación sugieren una tasa de abandono del 20\%, muy similar a la obtenida por Campbell et al. ${ }^{(21)}$, si bien la tasa en el caso del grupo psicoeducativo fue aún mayor (35\%). Es posible que esta diferencia pueda deberse a factores relacionados con la dinámica propia de cada hospital en los protocolos de atención a los enfermos, pero también es posible que los pacientes del grupo experimental tuvieran 
una mayor motivación, por lo que quizá debamos plantearnos si todo paciente está en condiciones de seguir una intervención psicológica, o de si todos los pacientes pueden seguir cualquier tipo de intervención. Sería interesante disponer de datos al respecto, aun cuando ésa sea otra línea de investigación, ya que lo que sí podemos decir es que, al menos en el momento del diagnóstico, el porcentaje de pacientes que desearían recibir atención psicooncológica es inferior al 30\% ${ }^{(41)}$.

\section{CONCLUSION}

Los datos de esta investigación indican que el MBSR produce una mejoría importante en el malestar emocional y en la calidad de vida, superior a la que puede lograrse con una intervención psicoeducativa. No obstante, no puede afirmarse que el MBSR deba ser la técnica de elección para todos los casos, pues no está claro que pueda ser seguida por cualquier tipo de paciente, al requerir una alta implicación por parte del mismo. Por otra parte, tampoco está claro que para niveles de alteración emocional no muy elevados (depresión leve) el MBSR sea mejor que otras técnicas que pueden ser más cómodas para el paciente. Es necesario seguir investigando para delimitar mejor los diferentes perfiles de paciente para los que puede estar indicado aplicar MBSR o bien otra de las técnicas actualmente disponibles en intervención psicooncológica.

AGRADECIMIENTOS: A la Fundació Joan Costa Romà del Consorci Sanitari de Terrassa por dotar económicamente el proyecto.

\section{REFERENCIAS BIBLIOGRÁFICAS}

1. Gurevich M, Devins GM, Rodin GM. Stress response syndrome and cancer:
Conceptual and assessment issues. Psychosomatic 2002;43:259-81. Doi: 10.1176/appi.psy.43.4.259

2. Koopman C, Butler LD, Classen C, GieseDavis J, Morrow GR, Westendorf J, et al. Traumatic stress symptoms among women with recently diagnosed primary breast. J Trauma Stress 2002;15:277-87. Doi:10.1023/A:1016295610660

3. Blake-Mortimer J, Gore-Felton C, Kimerling R, Turner-Cobb JM, Spiegel D. Improving the quality of life among patients with cancer: A review of the effectiveness of group psychotherapy. Eur J Cancer 1999;35:1581-6.

4. Rehse B, Pukrop R. Effects of psychosocial interventions on quality of life in adult cancer patients: A metaanalysis of 37 published controlled outcome studies. Patient Educ Couns 2003;50:179-86.

5. Newell SA, Sanson-Fisher RW, Savolainen NJ. Systematic review of psychological therapies for cancer patients: Overview and recommendations for future research. J Natl Cancer Inst 2002;94:558-84. Doi: 10.1093/jnci/94.8.558

6. Kabat-Zinn, J. An outpatient program in behavioural medicine for chronic pain patients based on the practice of mindfulness meditation: theoretical considerations and preliminary results. Gen Hosp Psychiatr 1982;4,33-47. Doi: 10.1016/01638343(82)90026-3

7. Martín-Asuero A, García-Banda G. The Mindfulness-based Stress Reduction program (MBSR) reduces stress-related psychological distress in healthcare professionals. Span J Psychol. 2010;13:897-905.

8. Kabat-Zinn, J. Four-year follow-uip of meditation based program for the selfregulation of chronic pain: Treatment outcomes and compliance. Clin J Pain 1987;2:159-73.

9. Kabat-Zinn J, Massion AO, Kristeller J, Peterson LG, Fletcher DE, Pbert L et al. Effectiveness of a meditation-based stress reduction program in the treatment of anxiety 
disorders. Am j Psychiatry 1992;149:93643.

10. Miller JJ, Fletcher K, Kabat-Zinn J. Threeyear follow-up and clinical implications of a mindfulness meditation-based stress reduction intervention in the treatment of anxiety disorders. Gen Hosp Psychiatry 1995;17:192-200. Doi: 10.1016/01638343(95)00025-M

11. Kaplan KH, Goldenberg DL, Galvin-Nadeau M. The impact of a meditation-based stress reduction program on fibromyalgia. Gen Hosp Psychiatry 1993;15:284-9. Doi: 10.1016/0163-8343(93)90020-O

12. Deepak KK, Manchanda SK, Maheshwari MC. Meditation improves clinicoelectroencephalographic measures in drugresistant epileptics. Biofeedback Self Regul 1994;19:25-40. Doi: 10.1007/ BF01720668

13. Schneider R, Staggers F, Alexander C, Sheppard W, Rainforth M, Kondwani K et al. A randomized controlled trial of stress reduction for hypertension in older african Americans. Hypertension 1995;26:820-7. Doi: 10.1161/01.HYP.26.5.820

14. Kabat-Zinn J, Wheeler E, Light T, Skillings A, Scharf MJ, Cropley TG et al. Influence of a mindfulness meditation-based stress reduction intervention on rates of skin clearing in patients with moderate to severe psoriasis undergoing phototherapy (UVB) and photochemotherapy (PUVA) Psychosom Med 1998;60:625-32 .

15. Bohlmeijer E, Prenger R, Taal E, Cuijpers P. The effects of mindfulness-based stress reduction therapy on mental health of adults with a chronic medical disease: A meta-analysis. J Psychosom Res 2010;68:539-44. Doi: 10.1016/j.jpsychores.2009.10.005

16. Speca M, Carlson L, Goodey E, Angen M. A randomized, waitlist controlled clinical trial: The effect of a Mindfulness-Based Stress Reduction program on mood and symptoms of stress in cancer outpatients. Psychosom Med 2000;62:613-22.
17. Ledesma, D., Kumano, H. Mindfulnessbased stress reduction and cancer: Metaanalysis. Psychooncology 2009;18:571-9. Doi: 10.1002/pon.1400

18. Ott MJ, Norris RL, Bauer-Wu SM. Mindfulness meditation for oncology patients: A discussion and critical review. Integrat Cancer Therapie 2006;5:98-108. Doi: 10.1177/1534735406288083

19. Shennan C, Payne S, Fenlon D. What is the evidence for the use of mindfulness-based interventions in cancer care? A review. Psychooncology 2011;20:681-97.

20. Birnie K, Garland SN, Carlson LE. Psychological benefits for cancer patients and their partners participating in mindfulnessbased stress reduction (MBSR). Psychooncology 2010;19:1004-9. Doi: 10.1002/ pon.1651

21. Campbell TS, Labelle LE, Bacon SL, Faris $\mathrm{P}$, Carlson LE. Impact of Mindfulness-Based Stress Reduction (MBSR) on attention, rumination and resting blood pressure in women with cancer: A waitlist-controlled study. J Behav Med 2012;35:262-71. Doi: 10.1007/s10865-011-9357-1

22. Van der Lee ML, Garssen B. Mindfulnessbased cognitive therapy reduces chronic cancer-related fatigue: A treatment study. Psychooncology 2012;21:264-72. Doi: 10.1002/pon.1890

23. Legancher CA, Johnson-Mallard V, PostWhite J, Moscoso MS, Jacobsen PB, Klein TW et al. Randomized controlled trial mindfulness-based stress reduction (MBSR) for survivors of breast cancer. Psychooncology 2009; 18:1231-72. Doi: 10.1002/ pon. 1529

24. Lengacher CA, Reich RR, Post-White J, Moscoso M, Shelton MM, Barta $M$, et al. Mindfulness based stress reduction in post-treatment breast cancer patients: An examination of symptoms and symptom clusters. J Behav Med 2012;35:86-94. Doi: 10.1007/s10865-011-9346-4

25. Rodriguez, B., Priede, A., Maeso, A., Arranz, H., Palao, A. Cambios psicológi- 
cos e intervenciones basadas en mindfulness para los supervivientes de cáncer. Psicooncologia 2011;8:7-20. Doi: 10.5209/ rev_PSIC.2011.v8.n1.1

26. Ledesma, D, Kumano H. Mindfulnessbased stress reduction and cancer: Metaanalysis. Psychooncology 2009;18:571-9. Doi: 10.1002/pon.1400

27. Wite-Janusek, L., Albuquerque, K., Rambo Chroniak, K., Chroniak, C., Durazo, R., Mathews, H. L. Effect of mindfulness based stress reduction on immune function, quality of life and coping in women newly diagnosed with early stage breast cancer. Brain Behav Immun 2008;22: 96981. Doi: 10.1016/j.bbi.2008.01.012

28. Kieviet-Stijnen, A., Visser, A, Garssen, B., Hudig, W. Mindfulness-based stress reduction training for oncology patients: Patients' appraisal and changes in wellbeing. Patient Educ Couns 2008;72: 43642. Doi: 10.1016/j.pec.2008.05.015

29. Brotto LA, Erskine $Y$, Carey $M$, Ehlen $T$, Finlayson S, Heywood $M$, et al. A brief mindfulness-based cognitive behavioral intervention improves sexual functioning versus wait-list control in women treated for gynecologic cancer. Gynecol Oncol 2012;125:320-5. Doi: 10.1016/j.ygyno.2012.01.035

30. Andersen SR, Würtzen $H$, Steding-Jessen $\mathrm{M}$, Christensen J, Andersen KK, Flyger $\mathrm{H}$ et al. Effect of mindfulness-based stress reduction on sleep quality: Results of a randomized trial among Danish breast cancer patients. Acta Oncol 2013;52:336-44. Doi: 10.3109/0284186X.2012.745948

31. Hoffman CJ, Ersser SJ, Hopkinson JB, Nicholls PG, Harrington JE, Thomas PW. Effectiveness of mindfulness-based stress reduction in mood, breast- and endocrine-related quality of life, and well-being in stage 0 to III breast cancer: a randomized, controlled trial. J Clin Oncol 2012;30:1335-42. Doi: 10.1200/JCO.2010.34.0331

32. Garland SN, Carlson LE, Antle MC, Samuels C, Campbell T. I-can sleep: Ra- tionale and design of a non-inferiority RCT of mindfulness-based stress reduction and cognitive behavioral therapy for the treatment of insomnia in cancer survivors. Contemp Clin Trials 2011;32:747-54. Doi: 10.1016/j.cct.2011.05.014

33. Carlson LE, Doll R, Stephen J, Faris P, Tamagawa $R$, Drysdale $E$ et al. randomized controlled trial of mindfulness-based cancer recovery versus supportive expressive group therapy for distressed survivors of breast cancer (MINDSET). J Clin Oncol 2013:31:3119-26. Doi: 10.1200/ JCO.2012.47.5210.

34. Henderson VP, Clemow L, Massion AO, Hurley TG, Druker S, Hébert JR. The effects of mindfulness-based stress reduction on psychosocial outcomes and quality of life in early-stage breast cancer patients: A randomized trial. Breast Cancer Res Treat 2012;131:99-109. Doi: 10.1007/s10549011-1738-1

35. Spielberger CD, Gorsuch RL, Lushene RE. STAI. Cuestionario de Ansiedad EstadoRasgo. Manual, $3^{\text {a }}$ ed. Madrid: TEA.

36. Beck AT, Beamesderfer A. Assesment of depression: The depression inventory. In: Pichot $\mathrm{P}$, editor. Moderrn problems in pharmacopsychiatry: Psychological measurements in psychopharmacology. Basel: Karger 1974.p.151-69.

37. Sanz J, García-Vera MP, Espinosa R, Fortún M, y Vázquez C. Adaptación española del Inventario para la Depresión de Beck II (BDI-II): 3. Propiedades psicométricas en pacientes con trastornos psicológicos. Clín Salud 2005;16:121-42.

38. Aaronson NK. Ahmedzai S, Bergman B, Bullinger M, Cull A, Duez NJ et al. The European Organization for the Research and Treatment of Cancer QLQ-C30: A quality of life instrument for use in international clinical trials in oncology. J Nat Cancer Instit 1993;85:365-76. Doi: 10.1093/ jnci/85.5.365

39. Arraras JI, Illarramendi JJ, Valerdi JJ. El cuestionario de Calidad de Vida de la 
EORTC, QLQ-C30. Estudio estadístico de validación con una muestra española. Rev Psic Salud 1995;7:13-33.

40. Ramachandra $\mathrm{P}$, Booth $\mathrm{S}$, Pieters $\mathrm{T}$, Vrotsou K, Huppert F.A. A brief selfadministered psychological intervention to improve well-being in patients with cancer: Results from a feasibility study.
Psychooncology 2009;18:1323-6. Doi: 10.1002/pon.1516

41. Leon C, Jovell E, Mirapeix R, Arcusa A, Blasco T. Factores predictores de solicitud de ayuda psicológica en los meses inmediatos al diagnóstico oncológico. Psicooncología 2012;9:289-98. Doi: 10.5209/rev_PSIC.2013.v9.n2-3.40898 